\title{
The Association of Dietary Polyphenol Intake with the Risk of Type 2 Diabetes: Tehran Lipid and Glucose Study
}

This article was published in the following Dove Press journal: Diabetes, Metabolic Syndrome and Obesity: Targets and Therapy

\author{
Zohreh Esfandiar' \\ Firoozeh Hosseini-Esfahani (D) \\ Parvin Mirmiran' \\ Emad Yuzbashian' \\ Fereidoun Azizi (iD) ${ }^{2}$ \\ 'Nutrition and Endocrine Research \\ Center, Research Institute for Endocrine \\ Sciences, Shahid Beheshti University of \\ Medical Sciences, Tehran, Iran; \\ ${ }^{2}$ Endocrine Research Center, Research \\ Institute for Endocrine Sciences, Shahid \\ Beheshti University of Medical Sciences, \\ Tehran, Iran
}

Correspondence: Parvin Mirmiran Nutrition and Endocrine Research Center, Research Institute for Endocrine Sciences, Shahid Beheshti University of Medical Sciences, Tehran, Iran

Tel +98 2l 22432500

Fax +98 21 22402463

Email mirmiran@endocrine.ac.ir

Firoozeh Hosseini-Esfahani

Nutrition and Endocrine Research

Center, Research Institute for Endocrine Sciences, Shahid Beheshti University of

Medical Sciences, Tehran, Iran

Email f.hosseini@sbmu.ac.ir
Purpose: The current study aimed to investigate the relationship between daily consumption of total polyphenol and its subclasses and the incidence of diabetes.

Materials and Methods: Eligible adults $(n=6,547)$ were chosen from among participants of the Tehran Lipid and Glucose Study (TLGS) with an average follow-up of 3.0 1.6 years. Dietary intakes were evaluated using a valid and reliable semi-quantitative food frequency questionnaire. Biochemical variables and anthropometrics were evaluated at baseline and follow-up examinations. Multivariate Cox proportional hazard regression models were used to estimate the development of type 2 diabetes mellitus (T2DM) in relation to total intake of polyphenol and its subclasses (flavonoids, phenolic acids, stilbenes, and lignans).

Results: This study was conducted on 2,882 men and 3,665 women, aged 41.3 \pm 14.6 and $39.0 \pm 13.4$ years, respectively. The number of participants with the new-onset T2DM was 253. Mean intake of total polyphenol was $346 \pm 245 \mathrm{mg} / 1000 \mathrm{kcal}$. Risk of type 2 diabetes decreased from quartiles 1 to 4 for total polyphenols (HR: 1.00, 0.37, 0.61, 0.50, $P_{\text {trend }}<0.01$ ), phenolic acids (HR: $1.00,0.57,0.49,0.45, P_{\text {trend }}<0.01$ ), and lignans (HR: $\left.1.00,0.67,0.61,0.60, P_{\text {trend }}<0.01\right)$, whereas non-significant results were found for flavonoids and stilbenes. This study suggests an inverse association between total intake of polyphenols, phenolic acids, and lignin, and the risk of T2DM.

Conclusion: These results emphasize the potential protective role of polyphenol rich food groups (especially fruits and vegetables) in the prevention of T2DM.

Keywords: polyphenols, flavonoids, phenolic acids, stilbenes, lignans, diabetes

\section{Introduction}

Type 2 diabetes mellitus (T2DM) is one of the most important public health problems in the world, and the best management for this preventable condition is dietary and lifestyle modifications. ${ }^{1}$ There is considerable evidence suggesting that natural polyphenols are effective in preventing and managing T2DM, probably due to their influence on the absorption, digestion and metabolism of glucose. ${ }^{2}$ Polyphenols are bioactive phytochemicals mostly found in plant foods, such as vegetables, fruit, legumes, cereal grains, wine, cocoa, coffee, and tea. Based on the biochemical structure, four major classes of polyphenols have been specified with different bioavailability: flavonoids (the class mostly studied), phenolic acids, stilbenes, and lignans. ${ }^{3}$

Research on the association between polyphenols and diabetes has increased since 2010. In vitro and in vivo studies suggest that polyphenol-rich food 
ameliorated glucose homeostasis. ${ }^{4,5}$ Several epidemiological studies and limited clinical studies reported that dietary polyphenols were associated with reduced incidence of diabetes, but these associations were controversial. ${ }^{6,8}$ Also, most observational and interventional studies focused on the intake of flavonoids and their subgroup; thus, there is limited data on other subclasses of polyphenol, even though they may be of equal importance. ${ }^{6,9,10}$

As far as we know, there is no study about the association between various classes of polyphenols and T2DM in the Middle East; hence, we aimed to prospectively evaluate the association between total intake of polyphenol and its subclasses with T2DM, in a group of Tehranian adults.

\section{Materials and Methods}

\section{Study Population}

Subjects of this cohort study were chosen from participants of the Tehran Lipid and Glucose Study (TLGS), a population-based prospective study carried out to identify the risk factors for non-communicable diseases in a sample of residents from District 13 of Tehran, the capital of Iran. ${ }^{11}$ The first examination survey was conducted from 1999 to 2001 on 15,005 subjects aged $\geq 3$ years, using the multistage stratified cluster random sampling technique, and follow-up examinations were performed every 3 years; 2002-2005 (survey 2), 2005-2008 (survey 3), 2008-2011 (survey 4), and 2012-2015 (survey 5) to identify risk factors or new developed diseases.

For the current study, of people who entered the 20052008, 2009-2011, and 2012-2015 surveys of TLGS, 11,509 subjects were randomly selected for dietary assessment. Of individuals who entered in surveys 3 and 4 , respectively, 1,856 and 6,568 subjects aged $\geq 18$ years were randomly selected for dietary assessment. A total of 8,424 adult women and men with available biochemical, anthropometric dietary and data were selected as the baseline population and followed until survey 5 (individuals entering at surveys 3 and 4 were followed, respectively, two times and once for the outcome measurements). Of these individuals, we excluded subjects with under- or over-reporting of energy intake $(<800$ or $\geq 4,200 \mathrm{kcal} /$ day) $(\mathrm{n}=496)$, pregnant or lactating women, and also those with prevalent T2DM ( $\mathrm{n}=959)$ at baseline. Finally, after excluding participants missing any follow-up data $(\mathrm{n}=422), 6,547$ subjects remained and entered for analysis (Figure 1).
Before taking part in this investigation, informed written consent was acquired from all participants. The study proposal was accepted by the Research Council of the Research Institute for Endocrine Sciences, Shahid Beheshti University of Medical Sciences. This study was conducted in accordance with the Declaration of Helsinki.

\section{Dietary Intake Measurements}

Dietary assessment was used by a valid and reliable 168item semi-quantitative food frequency questionnaire (FFQ); trained interviewers collected information on usual dietary intake, through face-to-face private interviews. ${ }^{12}$ The consumption frequency of each food item on a daily, weekly, or monthly basis was converted to daily intakes, and portion sizes were then converted into grams using household measures. The Iranian food composition table (FCT) is incomplete; therefore, the United States Department of Agriculture (USDA) FCT was used to analyze foods.

The polyphenol content of foods in our FFQ were obtained by using the Phenol-Explorer database (www. phenol-explorer.eu/contents). The total polyphenol value was calculated as the sum of four major subclasses of polyphenols as identified by chromatography. ${ }^{13}$

\section{Physical Activity}

Physical activity level was assessed using the Persiantranslated modifiable activity questionnaire (MAQ) with high reliability and moderate validity. ${ }^{14}$ Data on the time and frequency of light, moderate, high, and very hard intensity activities were obtained according to the list of common activities of daily life over the past year, and these activity data were transformed into metabolic equivalent-hours/week (Met/hours/week). ${ }^{15}$

\section{Blood Pressure and Anthropometric Measurements}

Systolic and diastolic blood pressures were measured twice (with a 30 second interval) in a sitting position after 15 minutes of rest.

The body weight was measured to the nearest $100 \mathrm{~g}$, using a digital scale (Seca 707), while subjects were minimally clothed and barefoot. Height was measured to the $0.5 \mathrm{~cm}$ by a tape measure, in standing position without shoes and with shoulders in normal alignment. Waist circumference (WC) was measured with a non-flexible tape meter without any pressure to the body's surface at the 


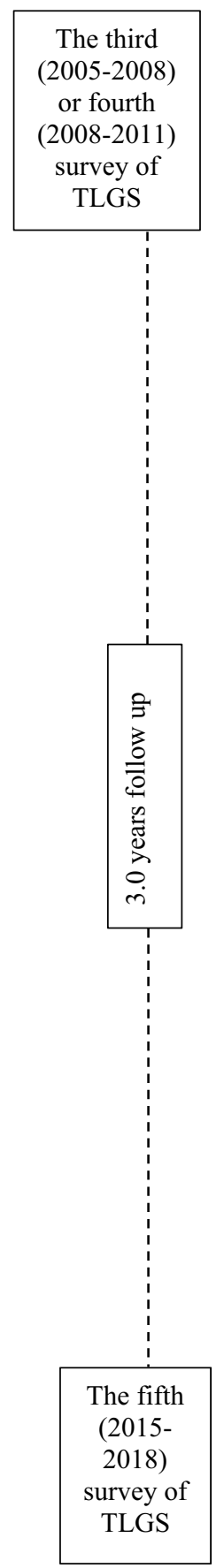

Figure I Outline of study participants.

level of the umbilicus and was taken at the end of a normal expiration, over light clothing. Measurements were recorded to the nearest $0.1 \mathrm{~cm}$.

\section{Laboratory Assays}

Blood samples were taken into vacutainer tubes at a time between 7:00 to 9:00 a.m, after 12-14 hours of overnight fasting. Samples were centrifuged within 30-45 minutes of
Total population of TLGS at baseline 2005-2008 (survey 3), (n=12523)

2008-2011(survey 4), (n=12823)

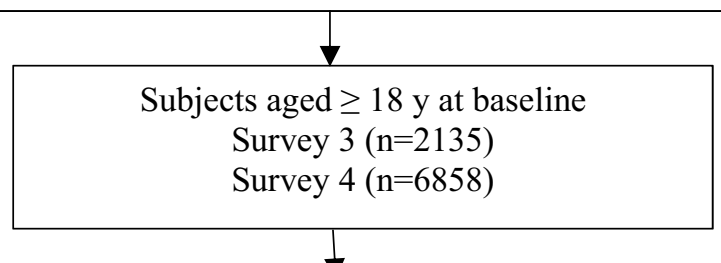

Completed dietary assessment at baseline Survey $3(\mathrm{n}=1856)$

Survey $4(\mathrm{n}=6568)$

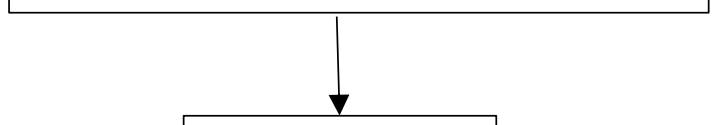

Free of type 2

diabetes at baseline $(\mathrm{n}=7465)$

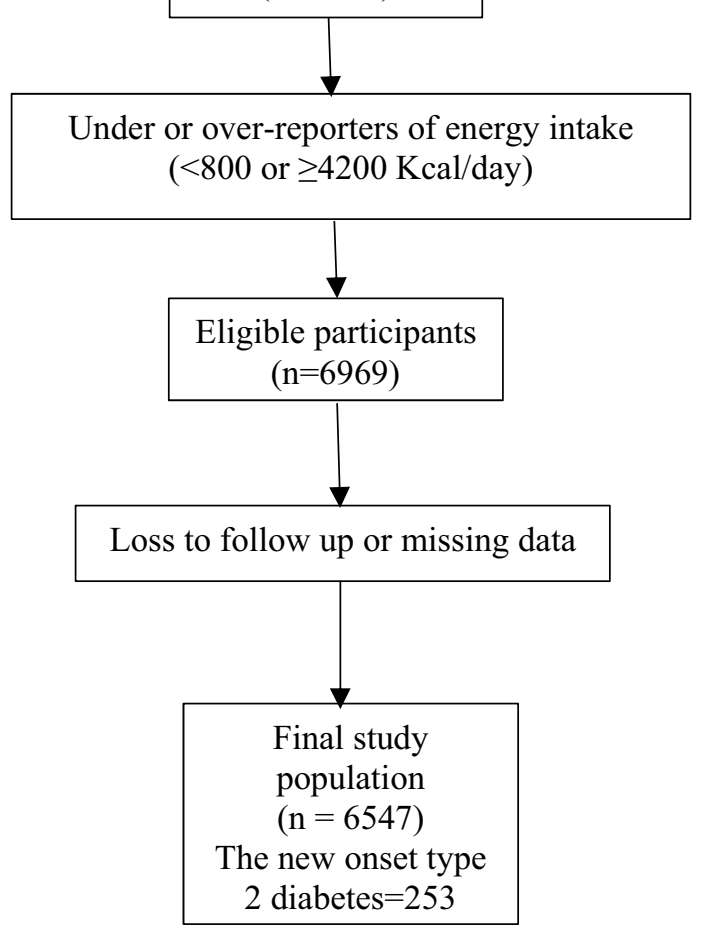

collection. All biochemical analyses were performed at the TLGS research laboratory, on the day of blood collection, and analyses of blood samples were conducted using a Selectra 2 auto-analyzer. Fasting blood glucose (FBS) concentration was measured on the day of blood collection by the enzymatic colorimetric method with the glucose oxidase technique. The standard 2-hour post-challenge blood glucose test was performed using oral 
administration of $82.5 \mathrm{~g}$ glucose monohydrate solution (equivalent to $75 \mathrm{~g}$ anhydrous glucose) for all individuals who were not on glucose-lowering drugs.

Triglyceride level was determined by enzymatic colorimetric tests using glycerol phosphate oxidase and triglyceride kits. HDL-C concentration was assessed after precipitation of the apolipoprotein B-containing lipoproteins with phosphotungstic acid. The inter- and intra-assay coefficients of variations were both $2.2 \%$ for glucose. For triglyceride, inter- and intra-assay coefficients of variations were $1.6 \%$ and $0.6 \%$, respectively. ${ }^{11}$

\section{Definitions}

Incidence of T2DM was defined as fasting plasma glucose concentrations $\geq 126 \mathrm{mg} / \mathrm{dL}$ or 2 -hour plasma glucose concentrations $\geq 200 \mathrm{mg} / \mathrm{dL}$, or treatment with anti-diabetic drugs. ${ }^{16}$ The diabetes risk score (DRS) was measured as follows: family history of T2DM ( 5 points) (a positive family history of diabetes was determined as having at least one parent or sibling with diabetes), SBP ( $\mathrm{mmHg}$ ) $<120$ (0 points), 120-140 (3 points), SBP $\geq 140$ (7 points); triglyceride/HDL-C: $<3.5$ (0 points), $\geq 3.5$ (3 points); waist-to-height ratio: $<0.54 \quad(0$ points $), \quad 0.54-0.59$

Table I Baseline Characteristics of Adult Participants of the Tehran Lipid and Glucose Study

\begin{tabular}{|c|c|c|c|c|c|}
\hline \multirow[t]{2}{*}{ Characteristics } & \multicolumn{4}{|c|}{ Total Polyphenol Consumption } & \multirow[t]{2}{*}{$P_{\text {trend }}{ }^{\pi}$} \\
\hline & QI & Q2 & Q3 & Q4 & \\
\hline Total polyphenol intake $(\mathrm{mg} / \mathrm{l}, 000 \mathrm{kcal} /$ day $)$ & $97.3 \pm 46.5^{\S}$ & $242 \pm 33.2$ & $364 \pm 40.8$ & $664 \pm 662$ & \\
\hline Baseline age (years) & $38.9 \pm 15.3$ & $38.4 \pm 13.4$ & $40.0 \pm 13.5$ & $4 \mid .4 \pm 13.6$ & 0.08 \\
\hline Women, \% (n) & $53.3(818)$ & $53.7(859)$ & $56.9(962)$ & $59.6(1,026)$ & 0.03 \\
\hline Current smokers (\%) & 14.7 & 11.2 & 10.7 & 13.1 & 0.80 \\
\hline Physical activity (MET/min/week) & $659 \pm 682$ & $687 \pm 888$ & $683 \pm 861$ & $616 \pm 650$ & 0.31 \\
\hline Education level $\geq 14$ years (\%) & 22.5 & 27.4 & 30.1 & 32.7 & 0.05 \\
\hline $\mathrm{BMI}\left(\mathrm{Kg} / \mathrm{m}^{2}\right)$ & $26.7 \pm 5.0$ & $26.8 \pm 4.3$ & $27.1 \pm 4.3$ & $27.2 \pm 4.6$ & 0.07 \\
\hline Waist circumference $(\mathrm{cm})$ & $89.9 \pm 13.3$ & $91.6 \pm 12.6$ & $91.6 \pm 12.1$ & $91.5 \pm 12.0$ & 0.36 \\
\hline SBP $(\mathrm{mmHg})$ & $112 \pm 16.8$ & $1 I I \pm 15.1$ & $112 \pm 16.0$ & $112 \pm 15.6$ & 0.30 \\
\hline $\mathrm{DBP}(\mathrm{mmHg})$ & $73.9 \pm 11.0$ & $75.3 \pm 10.5$ & $75.6 \pm 10.8$ & $75.2 \pm 10.3$ & 0.41 \\
\hline Total cholesterol (mg/dL) & $183 \pm 39.7$ & $183 \pm 38.2$ & $185 \pm 38.6$ & $186 \pm 36.2$ & 0.06 \\
\hline TG/HDL-ratio & $3.4 \pm 2.7$ & $3.1 \pm 2.6$ & $3.1 \pm 2.6$ & $3.0 \pm 2.4$ & 0.15 \\
\hline FBS (mg/dL) & $89.1 \pm 9.2$ & $92.2 \pm 8.4$ & $92.4 \pm 8.9$ & $92.5 \pm 8.7$ & 0.27 \\
\hline $2 \mathrm{~h}$ - plasma glucose $(\mathrm{mg} / \mathrm{dL})$ & $98.9 \pm 26.9$ & $100 \pm 26.2$ & $10 I \pm 26.1$ & $101 \pm 27.2$ & 0.15 \\
\hline Energy intake (kcal/day) & $2,156 \pm 709$ & $2,338 \pm 723$ & $2,452 \pm 688$ & $2,595 \pm 733$ & 0.03 \\
\hline Carbohydrate (\% of energy) & $57.3 \pm 8.1$ & $58.8 \pm 6.8$ & $59.6 \pm 6.8$ & $59.5 \pm 10.7$ & 0.20 \\
\hline Protein (\% of energy) & $13.9 \pm 3.0$ & $14.6 \pm 3.1$ & $14.8 \pm 3.4$ & $15.2 \pm 9.9$ & 0.06 \\
\hline Total fat (\% of energy) & $34.8 \pm 8.7$ & $33.4 \pm 6.9$ & $33.2 \pm 6.9$ & $33.4 \pm 24.0$ & 0.35 \\
\hline SFA (\% of energy) & $10.3 \pm 4.0$ & $9.9 \pm 2.8$ & $9.7 \pm 2.8$ & $10.1 \pm 21.4$ & 0.79 \\
\hline MUFA (\% of energy) & $10.7 \pm 3.0$ & $9.9 \pm 2.6$ & $9.9 \pm 2.7$ & $10.4 \pm 21.4$ & 0.87 \\
\hline PUFA (\% of energy) & $6.6 \pm 3.2$ & $5.9 \pm 1.9$ & $5.9 \pm 1.8$ & $6.4 \pm 2.1$ & 0.96 \\
\hline Fiber $(g / l, 000 \mathrm{kcal})$ & $17.2 \pm 8.4$ & $19.2 \pm 6.7$ & $19.7 \pm 7.0$ & $20.5 \pm 24.8$ & 0.09 \\
\hline Fruits (serving/day) & $2.1 \pm 1.7$ & $2.3 \pm 1.9$ & $2.7 \pm 2.2$ & $3.4 \pm 3.2$ & $<0.01$ \\
\hline Vegetables (serving/day) & $3.5 \pm 2.1$ & $4.0 \pm 2.2$ & $4.5 \pm 2.9$ & $5.0 \pm 3.2$ & 0.02 \\
\hline Legumes (serving/day) & $0.23 \pm 0.61$ & $0.44 \pm 0.47$ & $0.46 \pm 0.45$ & $0.5 I \pm 0.64$ & 0.16 \\
\hline Whole grains (serving/day) & $1.2 \pm 2.9$ & $1.9 \pm 2.3$ & $1.9 \pm 2.7$ & $1.9 \pm 2.6$ & 0.23 \\
\hline Refined grains (serving/day) & $9.3 \pm 5.2$ & $9.8 \pm 5.3$ & $9.6 \pm 4.8$ & $9.6 \pm 5.0$ & 0.64 \\
\hline Dairy products (serving/day) & $2.0 \pm 1.1$ & $2.1 \pm 1.1$ & $2.2 \pm 1.1$ & $2.2 \pm 1.1$ & 0.14 \\
\hline Red meat (serving/day) & $0.64 \pm 0.51$ & $0.62 \pm 0.64$ & $0.6 I \pm 0.5 I$ & $0.67 \pm 0.65$ & 0.42 \\
\hline Fish and poultry (serving/day) & $1.3 \pm 1.3$ & $1.4 \pm 1.4$ & $1.4 \pm 1.2$ & $1.5 \pm 1.5$ & 0.04 \\
\hline Sugar-sweetened soft drinks (mL/day) & $36.8 \pm 66.2$ & $49.0 \pm 78.1$ & $49.8 \pm 73.4$ & $54.9 \pm 77.0$ & 0.11 \\
\hline Nuts (serving/day) & $0.39 \pm 0.81$ & $0.44 \pm 0.74$ & $0.56 \pm 2.5 \mathrm{I}$ & $0.72 \pm 1.82$ & $<0.01$ \\
\hline Tea and coffee (mL/day) & $506 \pm 532$ & $46 I \pm 312$ & $604 \pm 332$ & $832 \pm 658$ & 0.06 \\
\hline
\end{tabular}

Notes: ${ }^{\S}$ Values are mean \pm SD unless otherwise listed; ${ }^{\top}$ Tests for trend across total polyphenol intake categories were performed by assigning each quartile its mean intake value and treating the variable as a linear regression.

Abbreviations: Q, quartiles of total polyphenol consumption; MET, metabolic equivalent; BMI, body mass index; MUFA, mono-unsaturated fatty acids; PUFA, polyunsaturated fatty acids; SFA, saturated fat. 
(6 points), $\geq 0.59$ (11 points); FBS $(\mathrm{mmol} / \mathrm{L}):<5.0(0$ points), $5.0-5.5$ (12 points), $5.6-6.9$ (33 points). ${ }^{17}$

\section{Statistical Analyses}

Statistical analyses were operated using the Statistical Package for Social Sciences (version 21.0; SPSS).

Baseline characteristics of the participants according to quartiles of the energy-adjusted daily intake of total polyphenols were computed using chi-square test for categorical variables and one-way ANOVA for continuous variables. Total polyphenols and its subclasses were adjusted for total energy intake $(\mathrm{mg} / 1,000 \mathrm{kcal})$. Considering a HR $=0.72,{ }^{9}$ power $=80 \%, \alpha=0.05$, and standardized cumulative incidence of $8.0 \%,{ }^{18}$ sample size was calculated. Multivariable Cox proportional hazard regression analyses were used to estimate the hazards ratio (HR) and 95\% confidence interval of incident T2DM. The occurrence of T2DM during the follow-up period was considered as dichotomous variables (yes/no) in the models. Total polyphenols, flavonoids, Phenolic acids, Stilbenes, and lignans intake were categorized into quartiles, the first quartile was considered as the reference. The median of each quartile was used as a continuous variable to estimate the overall trends of HRs across quartiles of total polyphenol and its subclasses in the Cox proportional hazard regression models. The confounders were chosen based on literature; also, each confounder was included in the univariable Cox regression model. A two-tailed $P$-value $<0.20$ was used for specifying inclusion in the model. The Cox regression models were adjusted for several potential confounders; the analyses were adjusted for sex, age,

Table 2 Hazard Ratios $(95 \% \mathrm{Cl}$ ) of Diabetes Across Energy-Adjusted Quartiles of Total Polyphenols (Flavonoids, Phenolic Acids, Stilbenes, and Lignans) in Adult Participants of The Tehran Lipid and Glucose Study ( $n=6,547)$

\begin{tabular}{|c|c|c|c|c|c|}
\hline \multirow[t]{2}{*}{ Variable } & \multicolumn{4}{|c|}{ Quartiles of Intake } & \multirow[t]{2}{*}{$P_{\text {trend }} \S$} \\
\hline & QI & Q2 & Q3 & Q4 & \\
\hline \multicolumn{6}{|l|}{ Total polyphenols } \\
\hline Median intake $(\mathrm{mg} /$ day & 85.8 & 244.7 & 361.7 & 575.4 & \\
\hline Diabetes incidence (\%) & 5.8 & 2.2 & 4.1 & 3.4 & \\
\hline Crude & I & $0.39(0.26-0.85)$ & $0.77(0.56-1.08)$ & $0.62(0.43-0.87)$ & $<0.01$ \\
\hline Model adjusted" & I & $0.37(0.25-0.56)$ & $0.61(0.44-0.85)$ & $0.50(0.35-0.72)$ & $<0.01$ \\
\hline \multicolumn{6}{|l|}{ Flavonoids } \\
\hline Median intake (mg/day) & 36.0 & 133.0 & 226.3 & $4 \mid 4.4$ & \\
\hline Diabetes incidence (\%) & 6.2 & 1.7 & 3.8 & 3.9 & \\
\hline Crude & I & $0.28(0.18-0.44)$ & $0.65(0.46-0.90)$ & $0.66(0.47-0.91)$ & 0.37 \\
\hline Model adjusted ${ }^{\pi}$ & I & $0.28(0.19-0.42)$ & $0.59(0.43-0.82)$ & $0.57(0.4 \mathrm{I}-0.80)$ & 0.08 \\
\hline \multicolumn{6}{|l|}{ Phenolic acids } \\
\hline Median intake (mg/day) & 4.7 & 80.6 & 116.4 & 183.4 & \\
\hline Diabetes incidence (\%) & 5.6 & 3.4 & 3.5 & 3 & \\
\hline Crude & I & $0.64(0.45-0.91)$ & $0.67(0.48-0.94)$ & $0.57(0.40-0.82)$ & $<0.01$ \\
\hline Model adjusted" & I & $0.57(0.40-0.80)$ & $0.49(0.35-0.68)$ & $0.45(0.32-0.64)$ & $<0.01$ \\
\hline \multicolumn{6}{|l|}{ Stilbenes } \\
\hline Median intake (mg/day) & 0.01 & 0.03 & 0.07 & 0.16 & \\
\hline Diabetes incidence (\%) & 4.4 & 3.4 & 4.0 & 3.6 & \\
\hline Crude & 1 & $0.78(0.55-I . I I)$ & $0.89(0.63-1.24)$ & $0.79(0.55-1.12)$ & 0.36 \\
\hline Model adjusted ${ }^{\pi}$ & I & $0.86(0.60-1.22)$ & $1.03(0.73-1.44)$ & $0.97(0.68-1.37)$ & 0.86 \\
\hline \multicolumn{6}{|l|}{ Lignans } \\
\hline Median intake (mg/day) & 1.6 & 3.6 & 5.6 & 9.1 & \\
\hline Diabetes incidence (\%) & 4.4 & 3.3 & 3.4 & 4.0 & \\
\hline Crude & 1 & $0.71(0.49-1.01)$ & $0.74(0.52-1.06)$ & $0.87(0.62-1.22)$ & 0.68 \\
\hline Model adjusted" & I & $0.67(0.47-0.96)$ & $0.61(0.43-0.87)$ & $0.60(0.43-0.85)$ & 0.02 \\
\hline
\end{tabular}

Notes: ${ }^{\S}$ Test for trend based on ordinal variable containing median value for each quartile; ${ }^{\Uparrow}$ Adjusted for age, sex, diabetes risk score, physical activity, fiber, total fat, and total energy intakes. 
physical activity (continuous), DRS, total fat (percentage of energy), dietary fiber $(\mathrm{g} / 1,000 \mathrm{Kcal})$, and total energy intake.

\section{Result}

This study was conducted on 6,547 subjects: 2,882 men aged $41.3 \pm 14.6$ years and 3,665 women aged $39.0 \pm 13.4$ years. Characteristics of the participants across quartiles of total polyphenol intakes are presented in Table 1. Total polyphenol intake was significantly higher among individuals in the upper quartile of energy intake compared to those in the lower quartile. Subjects in the higher quartiles of total polyphenol intake tended to consume more fruits, vegetables, fish and poultry, and nuts.

During a mean 3.0 \pm 1.6 years of follow-up, new-onset T2DM developed in 253 participants (3.9\%). The Cox proportional HRs for T2DM according to quartiles of total polyphenols and its four major classes are presented in Table 2.

After adjustment, risk of diabetes decreased from quartiles 1 to 4 for total polyphenols (HR: 1.00, 0.37, 0.61, $0.50, P_{\text {trend }}<0.01$ ) (Figure 2), phenolic acids (HR: 1.00, $0.57,0.49,0.45, P_{\text {trend }}<0.01$ ) (Figure 3 ), and lignans (HR: $1.00,0.67,0.61,0.60, P_{\text {trend }}=0.02$ ) (Figure 4), whereas non-significant results were found for flavonoids and stilbens (Table 2).

After splitting individuals based on under/normal weight $(\mathrm{BMI}<25)$ and overweight/obese $(\mathrm{BMI} \geq 25)$, the risk of T2DM decreased across the quartiles of total polyphenol intake in under/normal weight [HR (95\% CI): 1.00, 0.89 (0.36-2.18), 0.57 (0.20-1.56), 0.30 (0.09-1.01), $\left.P_{\text {trend }}=0.03\right]$ and overweight/obese [HR $(95 \% \mathrm{CI}): 1.00$, 0.33 (0.21-0.50), 0.63 (0.45-0.88), $0.53 \quad$ (0.37-0.88), $\left.P_{\text {trend }}<0.01\right]$.

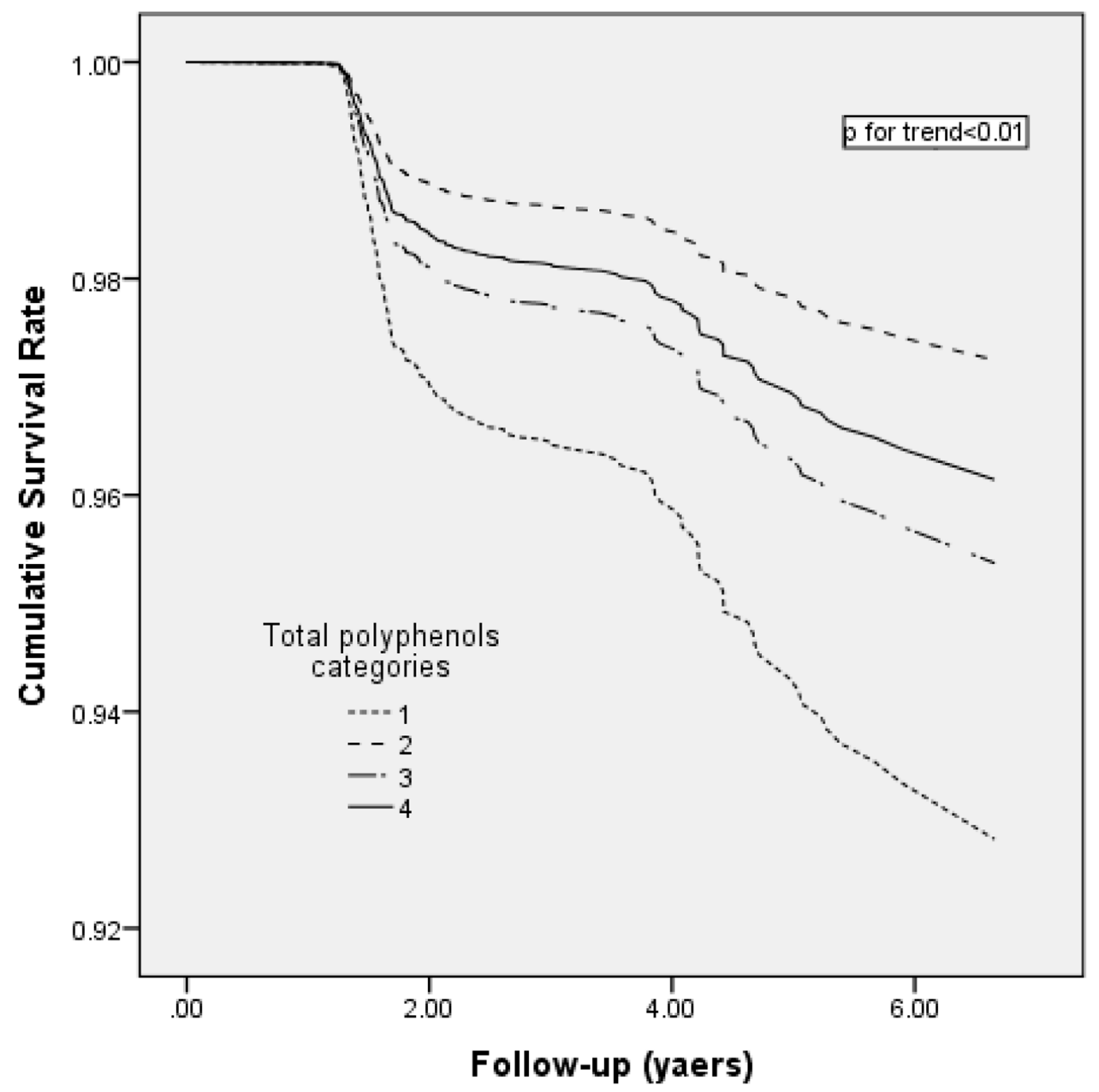

Figure 2 Multivariable-adjusted cumulative survival curves for incidence of type 2 diabetes mellitus according to total polyphenol categories. Multivariable-adjusted model included age, sex, diabetes risk score, physical activity, energy intake, fiber, and total fat intakes. 


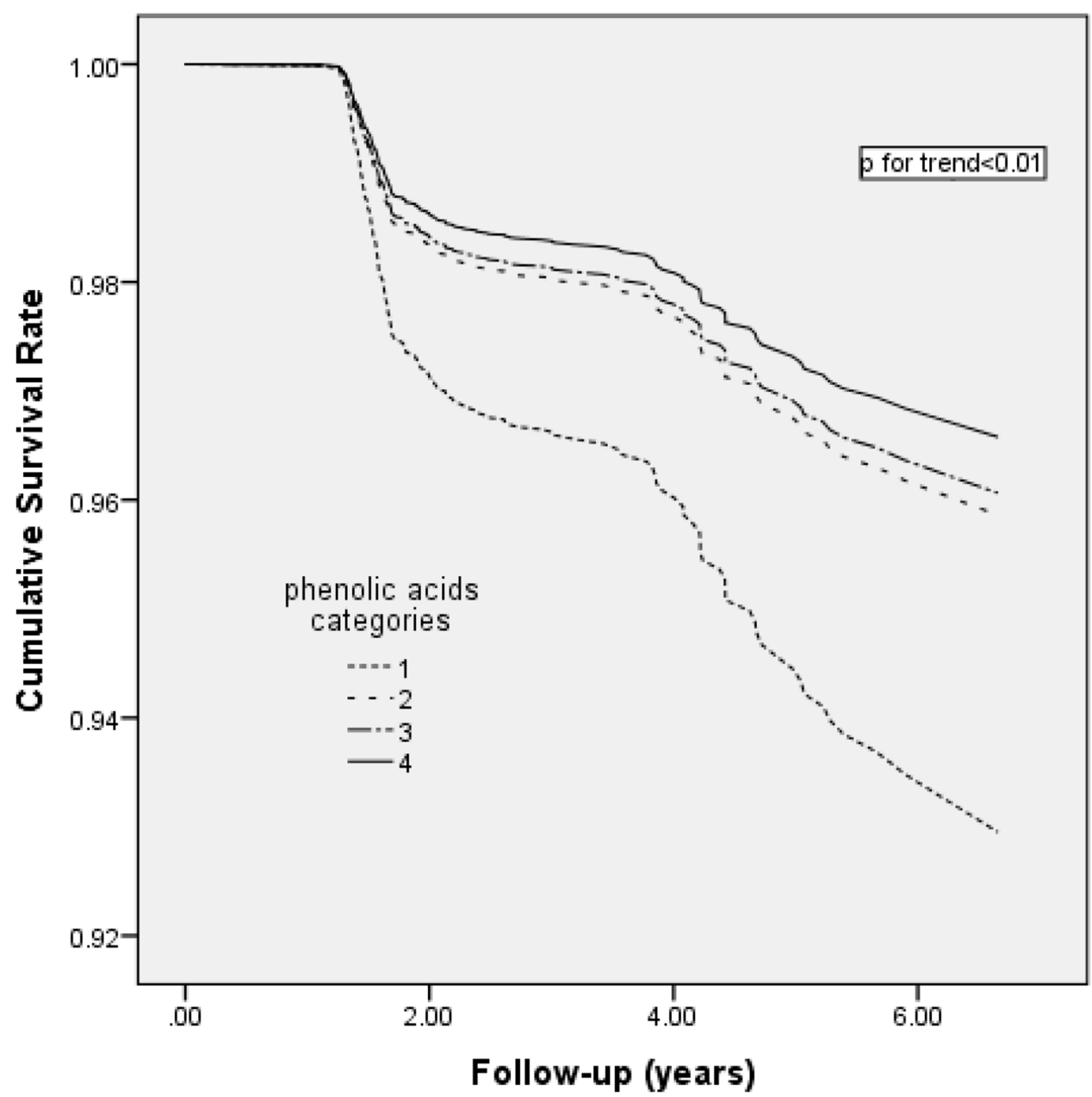

Figure 3 Multivariable-adjusted cumulative survival curves for incidence of type 2 diabetes mellitus according to phenolic acid categories. Multivariable-adjusted model included age, sex, diabetes risk score, physical activity, energy intake, fiber, and total fat intakes.

\section{Discussion}

Our results in this prospective cohort study suggested that a higher intake of total polyphenols, phenolic acids, and lignans were inversely associated with incidence of T2DM.

Although previous studies have reported the association between consumption of particular classes of polyphenols and diabetes, only two studies comprehensively evaluated the association between all polyphenol subclasses and incident $\mathrm{T} 2 \mathrm{DM} ;{ }^{9,10}$ an inverse relationship has been found between total polyphenols, stilbenes, and total flavonoids and incidence of T2DM, in the PREDIMED ${ }^{9}$ and HAPIEE $^{10}$ studies. Consistent with these results, total polyphenol intake in our population was inversely associated with T2DM, but no such association was observed between stilbene intake and T2DM in our study population. This contradiction could be due to the different stilbene intake; in our study, the median stilbenes intake in the last quartile
$(0.16 \mathrm{mg} /$ day $)$ was lower than that in the last tertile $(3.89$ $\mathrm{mg} /$ day) of the PREDIMED population. It is remarkable that the red wine intake (main source of stilbenes) was high in the PREDIMED population, whereas the amount of red wine consumption in our study population was almost zero. Because intake of stilbenes is found in such small quantities in this population, any protective effect of this polyphenol is unprovable at this level of intake.

There is no consensus on the anti-diabetic attributes of total flavonoids, the most consumed polyphenols. Consistent with our result, no significant association was found between total flavonoids intake and the risk of T2DM in four large prospective cohort studies. ${ }^{19,22}$ On the contrary, total flavonoids intake was inversely associated with T2DM in the PREDIMED cohort Study, ${ }^{9}$ and a meta-analysis study. ${ }^{6}$ This inconsistency can be due to measurement errors in dietary intakes and the genetic background of the study population. ${ }^{23}$ 


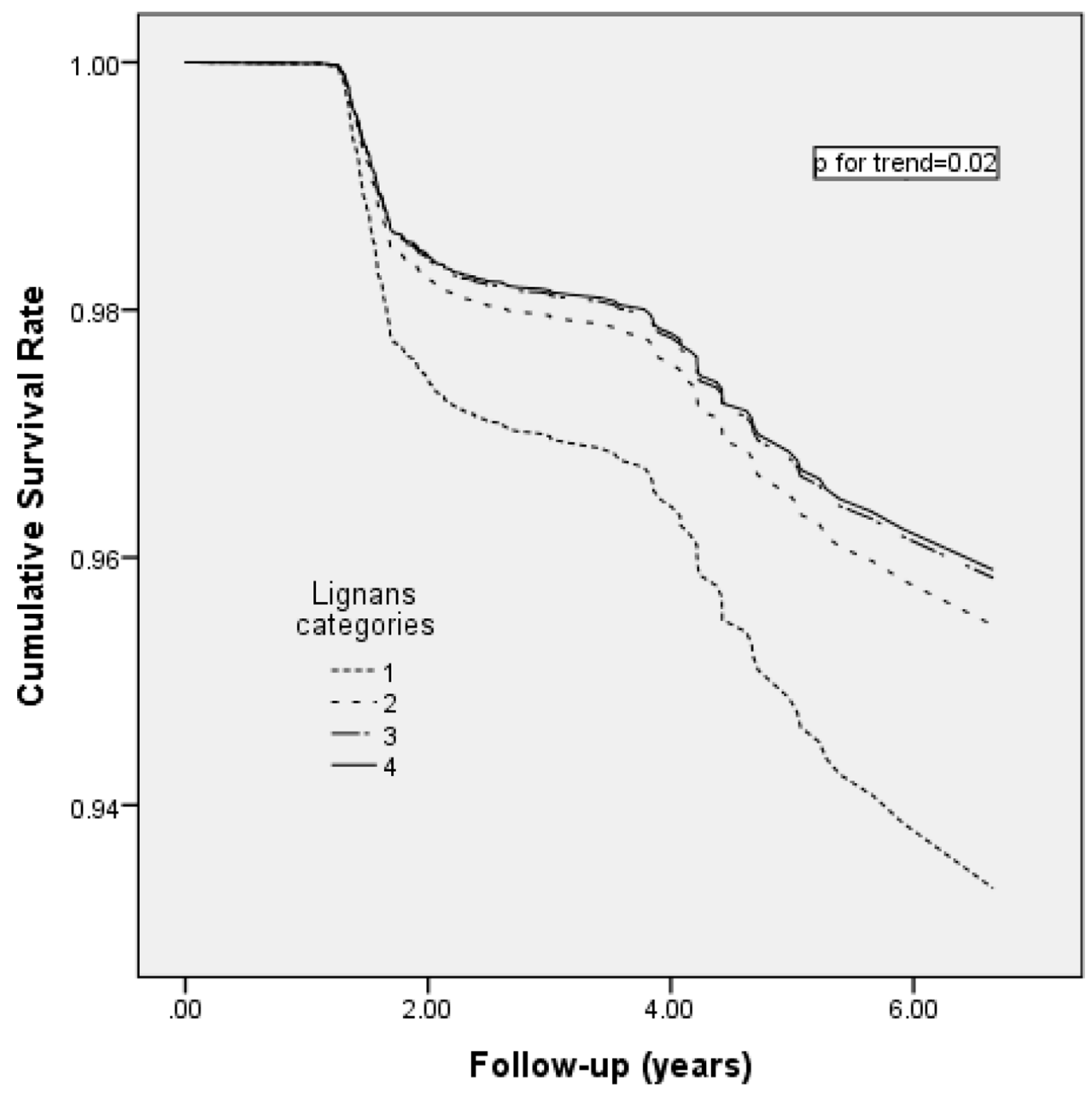

Figure 4 Multivariable-adjusted cumulative survival curves for incidence of type 2 diabetes mellitus according to lignan categories. Multivariable-adjusted model included age, sex, diabetes risk score, physical activity, energy intake, fiber, and total fat intakes.

Phenolic acids, a group of polyphenols mainly found in tea and coffee, had a strong inverse association with T2DM in our population and Eastern European populations, ${ }^{10}$ probably because tea consumption is higher in the Iranian population, and, consequently, the intake of phenolic acids from this source is greater. The anti-diabetic effects of tea have been revealed by previous studies; two meta-analyses reported that individuals drinking at least three ${ }^{24}$ or four ${ }^{25}$ cups of tea per day had lower incidence of T2DM, compared with lower intakes, althoughcontrasting findings have been published in another meta-analysis study. ${ }^{26}$

An inverse association was reported between lignans and T2DM in the present study. Sun et $\mathrm{al},{ }^{27}$ when investigating the relationship between urinary excretion of lignans (marker of total lignans intake) and the incidence of T2DM in the Nurses' Health Study cohort, found that urinary excretion of lignans is associated with a lower risk of T2DM in US women. In the European Prospective Study, ${ }^{28}$ however, the trend for an inverse association between lignin and T2DM was not significant. Lignans, which are one of the main types of phytoestrogens, have been hypothesized to improve glucose homeostasis by reducing the activity of essential enzymes related to plasma glucose. ${ }^{29}$

Due to the complex risk factors of T2DM and the various types of polyphenols, it is still indeterminate how dietary polyphenols impact T2DM. Although, some clinical researches have reported that the hypoglycemic effects of polyphenols may be due to prevention of carbohydrate digestion by inhibiting alpha-glucosidase and alpha-amylase, preventing glucose release from the liver, protecting beta-cells against glucose toxicity, and stimulating insulin secretion. ${ }^{30,31}$

The present study has important strengths. The evaluation of nutrient intake from various food sources provided a new vision into the relationship between nutrients and 
disease. The prospective design allowed the estimation of incident disease with less worry about reverse causality between nutrients and outcomes. The current study had its limitations too; since assessment of diet was implemented only at baseline, changes in dietary habits were not taken during follow-up. Nevertheless, change in dietary habits is unlikely during 3 years. The current data is based on food consumption report, while using multiple assessments of urine or blood measures of polyphenol metabolites over time could be a more reliable approach.

\section{Conclusion}

In conclusion, our study suggested an inverse association between total polyphenols, phenolic acids, and lignan intakes, and the risk of T2DM. These results emphasize the potential protective role of polyphenols rich diet (plant-based diets) in the prevention of T2DM.

\section{Acknowledgment}

The authors wish to acknowledge Dr. Forough Ghanbari for critical editing of the English grammar and syntax of the manuscript.

\section{Disclosure}

The authors report no conflicts of interest in this work.

\section{References}

1. Ley SH, Hamdy O, Mohan V, Hu FB. Prevention and management of type 2 diabetes: dietary components and nutritional strategies. Lancet. 2014;383(9933):1999-2007. doi:10.1016/S0140-6736(14)60613-9

2. Williamson G. Possible effects of dietary polyphenols on sugar absorption and digestion. Mol Nutr Food Res. 2013;57(1):48-57. doi:10. $1002 / \mathrm{mnfr} .201200511$

3. Manach C, Scalbert A, Morand C, Remesy C, Jimenez L. Polyphenols: food sources and bioavailability. Am J Clin Nutr. 2004;79(5):727-747. doi:10.1093/ajen/79.5.727

4. Kim Y, Keogh J, Clifton P. Polyphenols and glycemic control. Nutrients. 2016;8(1):17. doi:10.3390/nu8010017

5. Angelino D, Godos J. Fruit and vegetable consumption and health outcomes: an umbrella review of observational studies. Int J Food Sci Nutr. 2019;70(6):652-667.

6. Liu YJ, Zhan J, Liu XL, Wang Y, Ji J, He QQ. Dietary flavonoids intake and risk of type 2 diabetes: a meta-analysis of prospective cohort studies. Clin Nutr. 2014;33(1):59-63. doi:10.1016/j.clnu.20 13.03.011

7. Xiao JB, Hogger P. Dietary polyphenols and type 2 diabetes: current insights and future perspectives. Curr Med Chem. 2015;22(1):23-38. doi:10.2174/0929867321666140706130807

8. Cao H, Ou J, Chen L, et al. Dietary polyphenols and type 2 diabetes: human study and clinical trial. Crit Rev Food Sci Nutr. 2018;59:1-9.

9. Tresserra-Rimbau A, Guasch-Ferré M, Salas-Salvadó J, et al. Intake of total polyphenols and some classes of polyphenols is inversely associated with diabetes in elderly people at high cardiovascular disease risk. J Nutr. 2016;146:767-777.
10. Grosso G, Stepaniak U, Micek A, et al. Dietary polyphenol intake and risk of type 2 diabetes in the Polish arm of the Health, Alcohol and Psychosocial factors in Eastern Europe (HAPIEE) study. $\mathrm{Br} \mathrm{J}$ Nutr. 2017;118(1):60-68. doi:10.1017/S0007114517001805

11. Azizi F, Ghanbarian A, Momenan AA, et al. Prevention of noncommunicable disease in a population in nutrition transition: tehran lipid and glucose study phase II. Trials. 2009;10:5. doi:10.1186/17456215-10-5

12. Mirmiran P, Esfahani FH, Mehrabi Y, Hedayati M, Azizi F. Reliability and relative validity of an FFQ for nutrients in the Tehran lipid and glucose study. Public Health Nutr. 2010;13 (5):654-662. doi:10.1017/S1368980009991698

13. Perez-Jimenez J, Neveu V, Vos F, Scalbert A. Systematic analysis of the content of 502 polyphenols in 452 foods and beverages: an application of the phenol-explorer database. J Agric Food Chem. 2010;58(8):4959-4969. doi:10.1021/jf100128b

14. Momenan AA, Delshad M, Sarbazi N, Rezaei Ghaleh N, Ghanbarian A, Azizi F. Reliability and validity of the Modifiable Activity Questionnaire (MAQ) in an Iranian urban adult population. Arch Iran Med. 2012;15(5):279-282. doi:10.12155/AIM.007

15. Ainsworth BE, Haskell WL, Whitt MC, et al. Compendium of physical activities: an update of activity codes and MET intensities. Med Sci Sports Exerc. 2000;32(9 Suppl):S498-504. doi:10.1097/000 05768-200009001-00009

16. American Diabetes Association. Diagnosis and classification of diabetes mellitus. Diabetes Care; January, 2019. Available from: http:// care.diabetesjournals.org/content/42/Supplement_1/S1.

17. Bozorgmanesh M, Hadaegh F, Ghaffari S, Harati H, Azizi F. A simple risk score effectively predicted type 2 diabetes in Iranian adult population: population-based cohort study. Eur $J$ Public Health. 2011;21(5):554-559. doi:10.1093/eurpub/ckq074

18. Golozar A, Khalili D, Etemadi A, et al. White rice intake and incidence of type-2 diabetes: analysis of two prospective cohort studies from Iran. BMC Public Health. 2017;17(1):133. doi:10.11 86/s12889-016-3999-4

19. Knekt P, Kumpulainen J, Jarvinen R, et al. Flavonoid intake and risk of chronic diseases. Am J Clin Nutr. 2002;76(3):560-568. doi:10.10 93/ajcn/76.3.560

20. Song Y, Manson JE, Buring JE, Sesso HD, Liu S. Associations of dietary flavonoids with risk of type 2 diabetes, and markers of insulin resistance and systemic inflammation in women: a prospective study and cross-sectional analysis. $J$ Am Coll Nutr. 2005;24(5):376-384. doi:10.1080/07315724.2005.10719488

21. Nettleton JA, Harnack LJ, Scrafford CG, Mink PJ, Barraj LM, Jacobs JDR. Dietary flavonoids and flavonoid-rich foods are not associated with risk of type 2 diabetes in postmenopausal women. $J$ Nutr. 2006;136(12):3039-3045. doi:10.1093/jn/136.12.3039

22. Wedick NM, Pan A, Cassidy A, et al. Dietary flavonoid intakes and risk of type 2 diabetes in US men and women. Am J Clin Nutr. 2012;95(4):925-933. doi:10.3945/ajen.111.028894

23. Guasch-Ferre M, Merino J, Sun Q. Dietary polyphenols, Mediterranean diet, prediabetes, and Type 2 diabetes: a narrative review of the evidence. Oxid Med Cell Longev. 2017;2017:67 23931.

24. Yang WS, Wang WY, Fan WY, Deng Q, Wang X. Tea consumption and risk of type 2 diabetes: a dose-response meta-analysis of cohort studies. Br J Nutr. 2014;111(8):1329-1339. doi:10.1017/S00071145 13003887

25. Yang J, Mao QX, Xu HX, Ma X, Zeng CY. Tea consumption and risk of type 2 diabetes mellitus: a systematic review and meta-analysis update. BMJ Open. 2014;4(7):e005632. doi:10.1136/bmjopen-2014005632

26. Huxley R, Lee CM, Barzi F, et al. Coffee, decaffeinated coffee, and tea consumption in relation to incident type 2 diabetes mellitus: a systematic review with meta-analysis. Arch Intern Med. 2009;169 (22):2053-2063. doi:10.1001/archinternmed.2009.439 
27. Sun Q, Wedick NM, Pan A, et al. Gut microbiota metabolites of dietary lignans and risk of type 2 diabetes: a prospective investigation in two cohorts of U.S women. Diabetes Care. 2014;37(5):12871295. doi: $10.2337 / \mathrm{dc} 13-2513$

28. Zamora-Ros R, Forouhi NG, Sharp SJ, et al. The association between dietary flavonoid and lignan intakes and incident type 2 diabetes in European populations: the EPIC-InterAct study. Diabetes Care. 2013;36(12):3961-3970. doi:10.2337/dc13-0877

29. Talaei M, Pan A. Role of phytoestrogens in prevention and management of type 2 diabetes. World J Diabetes. 2015;6(2):271-283. doi:10.4239/wjd.v6.i2.271
30. Xie Y, Chen X. Structures required of polyphenols for inhibiting advanced glycation end products formation. Curr Drug Metab. 2013;14(4):414-431. doi:10.2174/1389200211314040005

31. Tsujita T, Shintani T, Sato H. alpha-Amylase inhibitory activity from nut seed skin polyphenols. 1. Purification and characterization of almond seed skin polyphenols. J Agric Food Chem. 2013;61 (19):4570-4576. doi:10.1021/jf400691q

\section{Publish your work in this journal}

Diabetes, Metabolic Syndrome and Obesity: Targets and Therapy is an international, peer-reviewed open-access journal committed to the rapid publication of the latest laboratory and clinical findings in the fields of diabetes, metabolic syndrome and obesity research. Original research, review, case reports, hypothesis formation, expert opinion and commentaries are all considered for publication. The manuscript management system is completely online and includes a very quick and fair peer-review system, which is all easy to use. Visit http://www.dovepress.com/testimonials.php to read real quotes from published authors. 